INPLASY

PROTOCOL

To cite: Li et al. Clinical effect of Tripterygium Glycosides in the treatment of connective tissue disease-related interstitial lung disease: Meta analysis. Inplasy protocol 202180028. doi: 10.37766/inplasy2021.8.0028

Received: 08 August 2021

Published: 08 August 2021

Corresponding author: Li Zifeng

1002581008@qq.com

Author Affiliation:

The First College of Clinical Medical Science, Three Gorges University/Yichang Central People's Hospital

Support: None.

Review Stage at time of this submission: Preliminary searches.

Conflicts of interest:

None declared.

\section{Clinical effect of Tripterygium Glycosides in the treatment of connective tissue disease-related interstitial lung disease: Meta analysis}

Li, ZF1; Fu, XL2; Yin, L3; Hou, XQ4; Chang, CY5.

Review question / Objective: Clinical effect of Tripterygium Glycosides in the treatment of connective tissue diseaserelated interstitial lung disease.

Condition being studied: Tripterygium wilfordii, as a Chinese medicine, has been widely used in the treatment of connective tissue disease in China, and has been gradually used in CTD-ILLD studies. We have not found any relevant meta-analysis yet.

Information sources: Databases: PubMed, Cochrane library, Web of Science, CNKI, wanfang database, Vip database. Contact authors, or grey papers, or conference papers.

INPLASY registration number: This protocol was registered with the International Platform of Registered Systematic Review and Meta-Analysis Protocols (INPLASY) on 08 August 2021 and was last updated on 08 August 2021 (registration number INPLASY202180028).

\title{
INTRODUCTION
}

Review question / Objective: Clinical effect of Tripterygium Glycosides in the treatment of connective tissue disease-related interstitial lung disease.
Condition being studied: Tripterygium wilfordii, as a Chinese medicine, has been widely used in the treatment of connective tissue disease in China, and has been gradually used in CTD-ILLD studies. We 
have not found any relevant meta-analysis yet.

\section{METHODS}

Participant or population: Patients with connective tissue disease-related interstitial lung disease (CTD-ILD).

Intervention: Glucocorticoids + tripterygium glycosides.

Comparator: Glucocorticoids.

Study designs to be included: RCT.

Eligibility criteria: American college of rheumatology classification of CTD-ILD.

Information sources: Databases: PubMed, Cochrane library, Web of Science, CNKI, wanfang database, Vip database. Contact authors, or grey papers, or conference papers.

Main outcome(s): Clinical efficacy.

Additional outcome(s): TCL, DLCO, $\mathrm{PaO} 2$ etc.

Data management: According to the inclusion and exclusion criteria, 2 researchers conducted preliminary screening of the retrieved literature, and used NOS (the newcastle-ottawa Scale) for quality evaluation and cross-checking. Third party evaluation of the controversial literature is conducted and unified through discussion. The two researchers extracted information related to the included study: author location, study design, publication time, sample size, intervention, outcome indicators, etc.

Quality assessment / Risk of bias analysis: Cochrane risk of bias assessment tool: seven entries were evaluated for each original literature included: random sequence generation (selection bias); Blinding allocation (selection bias); Blinding of all study participants and personnel (execution bias); Blinding of outcome assessment (observational bias); Completeness of outcome data (loss to follow-up bias); Selection of reports (reporting bias); Others. Each entry was judged into three categories, 'low risk', 'high risk' and 'unclear risk'.Grade of recommendation for each literature: Grade A: low level of bias, i.e. complete satisfaction of 4 and more entries with less potential for bias to occur; Grade B: moderate bias, i.e. 2 or 3 entries were fully met with moderate likelihood of bias;Level C: highly biased, 1 entry fully met or none met, with a high likelihood of bias.Finally, the results of the review of all included papers are shown in text, tables or figure legends.

Strategy of data synthesis: The metaanalysis was performed using Revman5.3 and STATA 15 software. Odds ratio (OR) and $95 \%$ confidence interval $(\mathrm{Cl})$ were selected from dichotomous data as the effect indexes of the meta-analysis.For continuous data, weighted mean difference (MD) or standardized mean difference (SMD) and $95 \% \mathrm{Cl}$ were used as effect indicators. Inter-study heterogeneity was determined by $\mathrm{X} 2$ test combined with 12 quantitative analysis.0.1, 12 \& $I t ; 50 \%$ thought inter-study heterogeneity was acceptable, and fixed effects model was used for meta-analysis.If $P<0.1,12$ \& gt; $50 \%$ believed that heterogeneity between studies was large, and random effects model was selected for meta-analysis when merging.Subgroup analysis or sensitivity analysis was used to find the source of heterogeneity, and Begg's test and Egger test were used to determine whether publication bias existed.

Subgroup analysis: Disease type, Drug dosing, Intervention duration etc.

Sensitivity analysis: The included studies were eliminated one by one, and the observational statistics were recombined to evaluate the stability of the conclusions.

Language: Chinese and English.

Country(ies) involved: China.

Keywords: CTD-ILLD, Connective Tissue Disease, Connective Tissue Disease. 
Contributions of each author:

Author 1 - Li Zifeng.

Author 2 - Fu Xiaolan.

Author 3 - Yin Long.

Author 4 - Hou Xiaoqiang.

Author 5 - Chang Caiyun. 